\title{
HARMONIC FUNCTIONS REPRESENTATION OF BESOV-LIPSCHITZ FUNCTIONS ON NESTED FRACTALS
}

\author{
Mats Bodin* and Katarzyna Pietruska-Pałuba ${ }^{\dagger}$ \\ Umeå University, Department of Ecology and Environmental Science \\ SE-901 87 Umeå, Sweden; mats.bodin@emg.umu.se \\ University of Warsaw, Institute of Mathematics \\ ul. Banacha 2, 02-097 Warsaw, Poland; kpp@mimuw.edu.pl
}

\begin{abstract}
R. S. Strichartz proposes a discrete definition of Besov spaces on self-similar fractals having a regular harmonic structure. In this paper, we characterize some of these Hölder-Zygmund and Besov-Lipschitz functions on nested fractals by means of the magnitude of the coefficients of the expansion of a function in a continuous piecewise harmonic base.
\end{abstract}

\section{Introduction}

The analysis on irregular sets such as the Sierpiński gasket has been developed since the late 80 's, starting with the pioneering works $[10,14]$. Also, the Brownian motion on fractals has been defined (see e.g. [1] for its definition, properties, and an extensive literature list). Both approaches yield the definition of the 'Laplace operator' $A$ on fractals. Having correctly defined the Laplacian, one can say that a function is 'harmonic' when it is annihilated by the Laplacian. However, such a definition of a harmonic function is very general and its properties are difficult to investigate.

On nested fractals (Sierpiński gasket-like), we are in a more favorable setting: their self-similar structure permits harmonic and 'locally harmonic' functions to be defined in an iterative manner. The Brownian motion, or more precisely the Dirichlet form associated with the Brownian motion, can be defined using discrete approximations as well. Such a discrete approach naturally yields the characteristics of the fractal, such as its Hausdorff dimension $d_{f}$ and its walk dimension $d_{w}$. They are recovered from the mass-scaling, length-scaling, and resistance-scaling factors, see (2.2) and (2.3) below.

Once the Dirichlet form $\mathcal{E}$ is defined together with its domain, then one has the variational definition of a harmonic function with prescribed boundary values: it is the unique function which minimizes $\mathcal{E}(f, f)$ among functions with given boundary values. Similar considerations can be carried out locally, which gives rise to the notion of an $m$-harmonic function (i.e. a 'locally harmonic', or 'piecewise harmonic'

doi:10.5186/aasfm.2012.3735

2010 Mathematics Subject Classification:

Key words: Besov spaces, Besov functions, Hölder functions, nested fractals, harmonic functions.

*This work was in part conducted while being employed as a fellow of the Marie Curie Transfer of Knowledge Programme SPADE2 (Deterministic and stochastic dynamics, fractals, turbulence) at the Institute of Mathematics of the Polish Academy of Sciences (IMPAN).

†Supported by the Polish Ministry of Science and Higher Education grant no. N N201 397837 (years 2009-2012). 
function), with values given at points of the $m$ th approximation of the fractal. Precise definitions are given below, in Section 2.2.2.

Since the geometry of nested fractals is decided after just one step of the iteration, the properties of harmonic functions are much easier to analyze with this approach. In particular, one can prove that harmonic functions are Lipschitz with respect to the so-called resistance metric on fractals, see [13].

Piecewise harmonic functions on nested fractals play a central role in analysis on fractals. For example, they enter the definition of the gradient and the derivative on fractals (see e.g. [14], [11], [20], [22]). On nested fractals, the derivative of a function at a given point is a harmonic function. This is not as strange as it looks: for a function on an interval, its derivative at a point is just a number, which can be identified with a direction in the plane, or with the tangent line passing through the point of its graph. In the one-dimensional setting, linear functions are exactly harmonic functions. We will use them to another goal: to provide a characterization of spaces of Lipschitz functions (Hölder-Zygmund, Besov-Lipschitz) on fractals. The spaces we will be working with are defined using intrinsic properties of the set $\mathcal{K}$ and do not really use the fact that they are embedded in $\mathbf{R}^{n}$. For their definition, we refer to [5], [21], and also to Section 2.2 below.

It is known (see e.g. [12]) that piecewise harmonic functions constitute a basis in the space of all continuous functions on a given nested fractal $\mathcal{K}$ : every continuous function $f$ on $\mathcal{K}$ can be written as a (countable) series $\sum c_{\xi} \psi_{\xi}(\cdot)$, with $\psi_{\xi}$ piecewise harmonic, and the series convergent uniformly and unconditionally. In this paper, we provide a characterization of Hölder-Zygmund and Besov-Lipschitz functions on $\mathcal{K}$ expressed in terms of the 'size' of their coefficients $c_{\xi}$ (Theorems 3.1, 3.2). A similar characterization as Theorem 3.2 for Besov spaces $B_{p q}^{s}(K)$ on nested fractals is presented in [9] and proved using atoms, and theorems for piecewise linear basis for certain subsets of $\mathbf{R}^{n}$ (in particular for the Sierpiński gasket) can be found in [7].

\section{Preliminaries}

We start with a description of the sets we will be working with, then we introduce the notion of harmonic and $m$-harmonic functions, and provide the definition of Hölder-Zygmund and Besov-Lipschitz spaces on nested fractals.

2.1. Nested fractals. Their definition goes back to Lindstrøm [15]. Suppose that $\phi_{1}, \ldots, \phi_{M}, M \geq 2$, are similitudes of $\mathbf{R}^{N}$ with common scaling factor $L>1$ (i.e. $\phi_{i}(x)=\frac{1}{L} U_{i}(x)+v_{i}, U_{i}$-isometries, $v_{i}$-vectors in $\left.\mathbf{R}^{N}\right)$. There exists a unique nonempty compact set (see [3], [15]) $\mathcal{K} \subset \mathbf{R}^{N}$ such that

$$
\mathcal{K}=\bigcup_{i=1}^{M} \phi_{i}(\mathcal{K}) .
$$

$\mathcal{K}$ is called the self-similar fractal generated by the family of similitudes $\phi_{1}, \ldots \phi_{M}$.

As all the similitudes $\phi_{i}$ are contractions, each of them has a unique fixed point $x_{i}$ (not necessarily distinct). Denote by $F$ the collection of those fixed points. A fixed point $x \in F$ is called an essential fixed point of the system $\phi_{1}, \ldots, \phi_{M}$ if there exists another fixed point $y \in F$ and two different transformations $\phi_{i}, \phi_{j}$ such that $\phi_{i}(x)=\phi_{j}(y)$. Denote by $V^{(0)}=\left\{v_{1}, \ldots, v_{r}\right\}$ the set of all essential fixed points of the system $\left\{\phi_{1}, \ldots, \phi_{M}\right\}$. 
For $A \subset \mathbf{R}^{N}$, let $\Phi(A)=\bigcup_{i=1}^{M} \phi_{i}(A)$ and $\Phi^{m}=\underbrace{\Phi \circ \cdots \circ \Phi}_{m \text { times }}$. Then we define 'the $m$-th level' vertices as

$$
V^{(m)}=\Psi^{m}\left(V^{(0)}\right), \quad m=1,2 \ldots
$$

$\left(V^{(m)}\right)_{m}$ is an increasing family of sets (it follows from the inclusion $V^{(0)} \subset \Phi\left(V^{(0)}\right)$ ). The vertices that are added in the $m$-th step will be denoted by $\mathcal{V}^{(m)}:=V^{(m)} \backslash V^{(m-1)}$, $m=1,2, \ldots$. For consistency, we set $\mathcal{V}^{(0)}=V^{(0)}$. Also, letting

$$
V^{(\infty)}=\bigcup_{m=0}^{\infty} V^{(m)}=\bigcup_{m=0}^{\infty} \mathcal{V}^{(m)}
$$

we have that $\mathcal{K}=\overline{V^{(\infty)}}$, the closure taken in the Euclidean topology of $\mathbf{R}^{N}$ (see [3], p. 120).

We impose certain conditions concerning the maps $\phi_{1}, \ldots, \phi_{M}$.

Condition 1. $\# V_{0} \geq 2$.

Condition 2. Open Set Condition. There exists an open, bounded, nonempty set $U \subset \mathbf{R}^{N}$ such that $\phi_{i}(U) \cap \phi_{j}(U)=\emptyset$ for $i \neq j$ and

$$
\Phi(U)=\bigcup_{i=1}^{M} \phi_{i}(U) \subset U .
$$

If the open set condition is satisfied, then the Hausdorff dimension of $\mathcal{K}$ is equal to (Th. 8.6 of $[3])$

$$
d_{f}=d_{f}(\mathcal{K})=\frac{\log M}{\log L} \leq N
$$

By $\mu$ we will denote the Bernoulli measure on $\mathcal{K}$, i.e. the restriction of the $x^{d_{f}}$ Hausdorff measure to $\mathcal{K}$, normalized so as to have $\mu(\mathcal{K})=1$. This measure is Ahlfors $d_{f}$-regular, i.e. measures of balls with radius $0<t \leq \operatorname{diam} \mathcal{K}$ and centers in $\mathcal{K}$ are comparable with $t^{d_{f}}$.

We collect the remaining notation that will be needed in the sequel in a single definition below. If $w=\left(i_{1}, \ldots, i_{n}\right)$, then we will abbreviate $\phi_{i_{1}} \circ \cdots \circ \phi_{i_{n}}$ to $\phi_{w}$.

Definition 2.1. Let $m \geq 0$ be given.

- An $m$-simplex is any set of the form $\phi_{w}(\mathcal{K})$, with $w=\left(i_{1}, \ldots, i_{m}\right)(m$-simplices are just scaled down copies of $\mathcal{K}$ ).

- Collection of all $m$-simplices will be denoted by $\mathcal{T}_{m}$.

- For an $m$-simplex $S=\phi_{w}(\mathcal{K})$, let $V(S)=\phi_{w}\left(V^{(0)}\right)$ be the set of its vertices.

- An $m$-cell is any of the sets $\phi_{w}\left(V^{(0)}\right)$.

- Two points $x, y \in V^{(m)}$ are called $m$-neighbors (denoted $x \stackrel{m}{\sim} y$ ), if they belong to a common $m$-cell.

Condition 3. Nesting. For each $m \geq 1$, and $S, T \in \mathcal{T}_{m}, S \cap T=V(S) \cap V(T)$.

Condition 4. Connectivity. Define the graph structure $E_{(1)}$ on $V^{(1)}$ as follows: we say that $(x, y) \in E_{(1)}$ if $x$ and $y$ are 1-neighbors. Then we require the graph $\left(V^{(1)}, E_{(1)}\right)$ to be connected. 
Condition 5. Symmetry. For $x, y \in V^{(0)}$, let $R_{x, y}$ be the reflection in the hyperplane bisecting the segment $[x, y]$. Then we stipulate that

$$
\forall_{i \in\{1, \ldots, M\}} \forall_{x, y \in V_{0}, x \neq y} \exists_{j \in\{1, \ldots, M\}} R_{x, y}\left(\phi_{i}\left(V^{(0)}\right)\right)=\phi_{j}\left(V^{(0)}\right) .
$$

Definition 2.2. (Nested fractal) The self-similar fractal $\mathcal{K}$, defined by (2.1) is called a nested fractal if the conditions $1-5$ are satisfied.

2.2. Functions on nested fractals. We will now describe notions necessary to define harmonic functions on nested fractals, as well as introduce the Hölder and Besov-Lipschitz spaces we will be working with.

2.2.1. The nondegenerate harmonic structure on $\mathcal{K}$. The material in this section is classical and follows [1]. See also [12].

An $r \times r$ real matrix $A=\left[a_{x, y}\right]_{x, y \in V^{(0)}}$ is called a conductivity matrix on $V^{(0)}$ if

(1) $\forall x \neq y, a_{x, y} \geq 0, a_{x, y}=a_{y, x}$;

(2) $\forall x, \sum_{y} a_{x, y}=0$.

We assume that $A$ is irreducible, i.e. the graph $\left\langle V^{(0)}, E_{(A)}\right\rangle$ is connected, where $E_{(A)}$ is the graph structure on $V^{(0)}$ induced by $A$, i.e. $V^{(0)} \times V^{(0)} \ni(x, y) \in E_{(A)}$ if and only if $a_{x, y}>0$.

For $A \subset \mathbf{R}^{N}$, let $C(A)$ denote the real-valued continuous functions on $A$. Any conductivity matrix on $V^{(0)}$ gives rise to a Dirichlet form on $C\left(V^{(0)}\right)$ : for $f \in C\left(V^{(0)}\right)$,

$$
\mathcal{E}_{A}^{(0)}(f, f)=\frac{1}{2} \sum_{x, y \in V^{(0)}} a_{x, y}(f(x)-f(y))^{2} .
$$

Two operations on Dirichlet forms are then defined, both relying on the geometry of $\mathcal{K}$.

1. Reproduction. For $f \in C\left(V^{(1)}\right)$ we let

$$
\widetilde{\mathcal{E}}_{A}^{(1)}(f, f)=\sum_{i=1}^{M} \mathcal{E}_{A}^{(0)}\left(f \circ \phi_{i}, f \circ \phi_{i}\right) .
$$

The mapping $\mathcal{E}_{A}^{(0)} \mapsto \widetilde{\mathcal{E}}_{A}^{(1)}$ is called the reproduction map and is denoted by $\mathcal{R}$.

2. Decimation. Given a symmetric form $\mathcal{E}$ on $C\left(V^{(1)}\right)$, denote its restriction to $C\left(V^{(0)}\right), \mathcal{E}_{V^{(0)}}$, as follows. Take $f: V^{(0)} \rightarrow \mathbf{R}$, then set

$$
\left.\mathcal{E}\right|_{V^{(0)}}(f, f)=\inf \left\{\mathcal{E}(g, g): g: V^{(1)} \rightarrow \mathbf{R} \text { and }\left.g\right|_{V^{(0)}}=f\right\} .
$$

This mapping is called the decimation map and will be denoted by $\mathcal{D} e$.

Let $\mathbf{G}$ be the symmetry group of $V^{(0)}$, i.e. the group of transformation generated by symmetries $R_{x, y}, x, y \in V^{(0)}$. Then we have ([15], [18]):

Theorem 2.1. Suppose $\mathcal{K}$ is a nested fractal. Then there exists a unique number $\rho=\rho(\mathcal{K})>1$ and a unique, up to a multiplicative constant, irreducible conductivity matrix $A$ on $V^{(0)}$, invariant under the action of $\mathbf{G}$, and such that

$$
(\mathcal{D} e \circ \mathcal{R})\left(\mathcal{E}_{A}^{(0)}\right)=\frac{1}{\rho} \mathcal{E}_{A}^{(0)}
$$

$A$ is called the symmetric nondegenerate harmonic structure on $\mathcal{K}$. It can be proven that all the nondiagonal entries of $A$ are positive. 
By analogy with the electrical circuit theory, $\rho$ is called the resistance scaling factor of $\mathcal{K}$. The number $d_{w}=d_{w}(\mathcal{K}) \stackrel{\operatorname{def}}{=} \frac{\log (M \rho)}{\log L}>1$ is called the walk dimension of $\mathcal{K}$.

2.2.2. The Dirichlet form and harmonic functions on $\mathcal{K}$. Suppose now that $A$ is given by Theorem 2.1. Define $\mathcal{E}^{(0)}=\mathcal{E}_{A}^{\langle 0\rangle}$, then let

$$
\widetilde{\mathcal{E}}^{(m)}(f, f)=\sum_{w=\left(i_{1}, \ldots, i_{m}\right)} \mathcal{E}^{(0)}\left(f \circ \phi_{w}, f \circ \phi_{w}\right)
$$

and further

$$
\mathcal{E}^{(1)}(f, f)=\rho \widetilde{\mathcal{E}}^{(1)}(f, f) ; \quad \mathcal{E}^{(m)}(f, f)=\rho^{m} \widetilde{\mathcal{E}}^{(m)}(f, f) .
$$

Multiplication by $\rho$ in every step makes the sequence $\mathcal{E}^{\langle m\rangle}$ nondecreasing, so we obtain that: for every $f: V^{(\infty)} \rightarrow \mathbf{R}$, and every $m=0,1,2, \ldots$ one has

$$
\mathcal{E}^{(m)}(f, f) \leq \mathcal{E}^{(m+1)}(f, f) .
$$

Set $\widetilde{\mathcal{D}}=\left\{f: V^{(\infty)} \rightarrow \mathbf{R}: \sup _{m} \mathcal{E}^{(m)}(f, f)<\infty\right\}$ and for $f \in \widetilde{\mathcal{D}}$

$$
\widetilde{\mathcal{E}}(f, f)=\lim _{m \rightarrow \infty} \mathcal{E}^{(m)}(f, f),
$$

and further $\mathcal{D}=\left\{f \in C(\mathcal{K}):\left.f\right|_{V^{(\infty)}} \in \widetilde{\mathcal{D}}\right\}, \mathcal{E}(f, f)=\widetilde{\mathcal{E}}\left(\left.f\right|_{V^{(\infty}},\left.f\right|_{V^{(\infty)}}\right)$ for $f \in \mathcal{D}$. $(\mathcal{E}, \mathcal{D})$ is a regular Dirichlet form on $L^{2}(\mathcal{K}, \mu)$, which agrees with the group of local symmetries of $\mathcal{K}$.

There are several possible ways of defining a harmonic (and an $m$-harmonic) function on $\mathcal{K}$. We choose the following one.

Definition 2.3. Suppose $f: V^{(0)} \rightarrow \mathbf{R}$ is given. Then $h \in \mathcal{D}(\mathcal{E})$ is called harmonic on $\mathcal{K}$ with boundary values $f$, if $\mathcal{E}(h, h)$ minimizes the expression $\mathcal{E}(g, g)$ among all $g \in \mathcal{D}(\mathcal{E})$ such that $\left.g\right|_{V^{(0)}}=f$.

Similarly, when $f: V^{(m)} \rightarrow \mathbf{R}$ is given $(m \geq 1)$, then $h \in \mathcal{D}(\mathcal{E})$ is called $m$ harmonic with boundary values $f$, if $\mathcal{E}(h, h)$ minimizes the expression $\mathcal{E}(g, g)$ among all $g \in \mathcal{D}(\mathcal{E})$ such that $\left.g\right|_{V^{(m)}}=f$.

Denote by $H_{m}$ the space of all $m$-harmonic functions on $\mathcal{K}$. For $f \in C(\mathcal{K})$, let $S_{m} f$ be the $m$-harmonic function that agrees with $f$ on $V^{(m)}$.

Definition 2.4. Suppose $\xi \in V^{(\infty)}$, and let $m$ be the unique number such that $\xi \in \mathcal{V}^{(m)}$. Then $\psi_{\xi}$ is, by definition, the unique $m$-harmonic function whose values on $V^{(m)}$ are given by

$$
\psi_{\xi}(\eta)= \begin{cases}1, & \eta=\xi \\ 0, & \eta \neq \xi\end{cases}
$$

2.2.3. Harmonic representation of continuous functions on $\mathcal{K}$. The collection of functions $\left\{\psi_{\xi}: \xi \in V^{(m)}=\bigcup_{i=0}^{m} \mathcal{V}^{(i)}\right\}$ constitutes a linear basis in $H_{m}$; one can write

$$
S_{m} f=\sum_{i=0}^{m} \sum_{\xi \in \mathcal{V}^{(m)}} c_{\xi} \psi_{\xi},
$$

where the coefficients $c_{\xi}$ are given by $c_{\xi}=f(\xi)-S_{m-1} f(\xi)$ (we set $S_{-1} \equiv 0$ ). 
It is not hard to see (cf. e.g. [1], p. 99, proof of Th. 7.14, or [12]), that for $f \in C(\mathcal{K}), S_{m} f \rightarrow f$ uniformly as $m \rightarrow \infty$. It follows that every continuous function on $\mathcal{K}$ admits an expansion

$$
f(x)=\sum_{i=0}^{\infty} \sum_{\xi \in \mathcal{V}^{(i)}} c_{\xi} \psi_{\xi}(x),
$$

with the series uniformly convergent in $\mathcal{K}$.

2.2.4. Hölder functions on $\mathcal{K}$. Following Strichartz (see [21], Def. 7.1), we define the Hölder-Zygmund spaces on $\mathcal{K}$ as follows.

Definition 2.5. Suppose $\alpha \in(0,1)$. Then $f \in \Lambda_{\alpha}^{(1)}(\mathcal{K})$ if and only if $f \in C(\mathcal{K})$ and

$$
\exists M \forall m \geq 0 \forall x \stackrel{m}{\sim} y \quad|f(x)-f(y)| \leq M L^{-\alpha m\left(d_{w}-d_{f}\right)} .
$$

It is known that (see [21], the discussion following Def. 7.1), that functions from $\Lambda_{\alpha}^{(1)}(\mathcal{K})$ are precisely those that satisfy $|f(x)-f(y)| \leq C R(x, y)^{\alpha}$, where $R(x, y)$ is the resistance metric on $\mathcal{K}$. On some fractals such as the Sierpiński gasket the resistance metric $R(x, y)$ is equivalent to $|x-y|^{d_{w}-d_{f}}$ (where $|x-y|$ denotes the Euclidean distance).

2.2.5. Besov-Lipschitz functions on $\mathcal{K}$. For the standard Sierpiński gasket $\mathcal{G}$ in $\mathbf{R}^{2}$, recall that the $L=2, M=3, d_{f}=\frac{\log 3}{\log 2}, d_{w}=\frac{\log 5}{\log 2}, \rho=\frac{5}{3}=L^{d_{w}-d_{f}}$. Let $d=\frac{d_{f}}{d_{w}-d_{f}}=\frac{\log 3}{\log 5-\log 3}$. The spaces $\left(\Lambda_{\alpha}^{p, q}\right)^{(1)}(\mathcal{G})$ were defined by Strichartz in [21] for $\frac{d}{p}<\alpha \leq \frac{d}{p}+\alpha_{1}$, where $\alpha_{1}=\frac{1}{d_{w}-d_{f}}$, and consist of those continuous functions on the gasket, for which

$$
\left\|\left(\left(2^{d_{w}-d_{f}}\right)^{m \alpha} \delta_{m, p}(f)\right)_{m}\right\|_{q}=\left\|\left(\rho^{m \alpha} \delta_{m, p}(f)\right)_{m}\right\|_{q}<\infty,
$$

where

$$
\delta_{m, p}(f)= \begin{cases}\left(2^{-m d_{f}} \sum_{x \stackrel{m}{\sim} y}|f(x)-f(y)|^{p}\right)^{\frac{1}{p}} & \text { if } p<\infty, \\ \sup \{|f(x)-f(y)|: x \stackrel{m}{\sim} y\} & \text { if } p=\infty .\end{cases}
$$

It is necessary to restrict the definition to continuous functions because the norm of these spaces is defined for a countable dense subset of the Sierpinski gasket. The condition $\alpha>d / p$ ensures that the functions are Lipschitz continuous. The relation $x \stackrel{m}{\sim} y$ is the neighboring relation on the $m$-th approximation of the gasket $V^{(m)}$, as described in Def. 2.1. In this particular case, if $x \stackrel{m}{\sim} y$, then $|x-y|=\frac{1}{2^{m}}$.

We can clearly extend this definition to nested fractals: supposing $f \in C\left(V^{(\infty)}\right)$, we put

$$
\delta_{m, p}(f)= \begin{cases}\left(L^{-m d_{f}} \sum_{x \rightarrow y}|f(x)-f(y)|^{p}\right)^{\frac{1}{p}} & \text { if } p<\infty \\ \sup \{|f(x)-f(y)|: x \stackrel{m}{\sim} y\} & \text { if } p=\infty .\end{cases}
$$

And we have:

Definition 2.6. Let $1 \leq p, q \leq \infty$ are given and let $\alpha>\frac{d}{p}$. We say that the function $f \in C(\mathcal{K})$ belongs to the space $\left(\Lambda_{\alpha}^{p, q}\right)^{(1)}(\mathcal{K})$ if

$$
\|f\|_{p, q, \alpha}:=\left\|\left(L^{\left(d_{w}-d_{f}\right) \alpha m} \delta_{m, p}(f)\right)_{m}\right\|_{q}<\infty .
$$


The norm in this space is given by

$$
\|f\|_{\Lambda_{\alpha}^{p, q}}=\|f\|_{p}+\|f\|_{p, q, \alpha} .
$$

Remark 2.1. One can naturally extend the "O-level" conductivities $a_{x, y}$ (given for $\left.x, y \in V^{(0)}\right)$ to $m$-th level conductivities. Suppose $x \stackrel{m}{\sim} y$, i.e. $x$ and $y$ belong to a common $m$-cell $\phi_{i_{1}} \circ \cdots \circ \phi_{i_{m}}\left(V^{0)}\right)$. Therefore we can uniquely determine two distinct vertices $v_{1}, v_{2} \in V^{(0)}$ such that $x=\phi_{i_{1}} \circ \cdots \circ \phi_{i_{m}}\left(v_{1}\right)$ and $y=\phi_{i_{1}} \circ \cdots \circ \phi_{i_{m}}\left(v_{2}\right)$. We set

$$
a_{x, y}^{(m)}=a_{v_{1}, v_{2}} .
$$

It would be more natural to take into account these $m$-level conductivities in the definition of $\delta_{m, p}$, i.e. to replace $|f(x)-f(y)|^{p}$ by $a_{x, y}^{p / 2}|f(x)-f(y)|^{p}$. But since the matrix $A$ related to the nondegenerate harmonic structure has nonzero off-diagonal entries, it would not change the class of functions in $\left(\Lambda_{\alpha}^{p, q}\right)^{(1)}(\mathcal{K})$, and the norms would be equivalent.

\section{Harmonic functions and Besov-Lipschitz spaces}

Another possible basis in $C(\mathcal{K})$, and historically the one first considered, is that consisting of "piecewise linear" functions on $\mathcal{K}$. On the Sierpiński gasket $\mathcal{G}$ for example, one have an expansion of continuous function, similar to the one previously described (2.6), in a series of piecewise linear functions: "piecewise linear" functions $\eta_{\xi}$ will replace the harmonic functions $\psi_{\xi}$ in $(2.6)$ : for $f \in C(\mathcal{G})$, we would have

$$
f(x)=\sum_{m=0}^{\infty} \sum_{\xi \in \mathcal{V}^{(m)}} d_{\xi} \eta_{\xi}(x),
$$

with the series uniformly convergent. Here $d_{\xi}$ are coefficients defined analogously to $c_{\xi}$. For details, see [7]. That paper dealt with certain subsets of $\mathbf{R}^{n}$, including e.g. the Sierpiński gasket (and also the Sierpiński carpet!), and function spaces $\operatorname{Lip}(\beta, p, q)$ on those sets. For their definition we refer e.g. to [8] or [5]. Since this definition is irrelevant for our purposes, we omit it.

The spaces $\operatorname{Lip}(\beta, p, q)$ are closely related to Strichartz's $\left(\Lambda_{\alpha}^{p, q}\right)^{(1)}$ spaces: it is proven in [2] that $\left(\Lambda_{\alpha}^{p, q}\right)^{(1)}=\operatorname{Lip}(\beta, p, q)$ for nested fractals (see [16] for a definition), where $\alpha$ and $\beta$ are related through the relation $\beta=\alpha\left(d_{w}-d_{f}\right)$. The authors of [7] have established a characterization of spaces $\operatorname{Lip}(\beta, p, q)$ on those subsets on $\mathbf{R}^{N}$ that admit a proper triangulation: a function $f \in C(\mathcal{K})$ belongs to $\operatorname{Lip}(\beta, p, q)$ if and only if its coefficients of its expansion in the piecewise linear basis $\left(\eta_{\xi}\right)$ are not too big (the precise condition is given in [7]). Similar considerations are carried over in [2] also for the Hölder-Zygmund spaces $\Lambda_{\alpha}^{(1)}(\mathcal{K})$ (again, under appropriate restraints concerning the set $\mathcal{K}$ ).

In what follows, we will give similar characterizations for the Hölder-Zygmund and Besov-Lipschitz spaces on $\mathcal{K}$, employing the piecewise-harmonic basis.

Before we start, we formulate and prove two lemmas, concerning the harmonic functions on the fractal.

Lemma 3.1. For all $x, y \in \mathcal{K}$, and $i \geq 0$, we have that

$$
\#\left\{\xi \in \mathcal{V}^{(i)}: \psi_{\xi}(x) \neq \psi_{\xi}(y)\right\} \leq 2 r,
$$

where $r$ is the number of essential fixed points. 
Proof. If $z \in \Delta \in \mathcal{T}_{i}$, then $\psi_{\xi}(z)=0$ unless $\xi \in V(\Delta)$, i.e. $\xi$ is a vertex of the $i$-simplex $\Delta$, which have $r$ vertices. Hence $\#\left\{\xi \in \mathcal{V}^{(i)}: \psi_{\xi}(z) \neq 0\right\} \leq r$ for all $z \in \mathcal{K}$. We get that $\#\left\{\xi \in \mathcal{V}^{(i)}: \psi_{\xi}(x) \neq \psi_{\xi}(y)\right\} \leq \#\left\{\xi \in \mathcal{V}^{(i)}: \psi_{\xi}(x) \neq 0\right.$ or $\left.\psi_{\xi}(y) \neq 0\right\} \leq$ $\leq \#\left\{\xi \in \mathcal{V}^{(i)}: \psi_{\xi}(x) \neq 0\right\}+\#\left\{\xi \in \mathcal{V}^{(i)}: \psi_{\xi}(y) \neq 0\right\} \leq 2 r$.

Lemma 3.2. Let $f \in C(\mathcal{K})$ and $\xi \in \mathcal{V}^{(1)}$. Then

$$
S_{0} f(\xi)=f\left(v_{1}\right) \psi_{v_{1}}(\xi)+\cdots+f\left(v_{r}\right) \psi_{v_{r}}(\xi) .
$$

Moreover, for all $x \in \mathcal{K}$ one has

$$
\psi_{v_{1}}(x)+\cdots+\psi_{v_{r}}(x)=1 .
$$

Proof of the lemma. The function $\phi(\cdot)=f\left(v_{1}\right) \psi_{v_{1}}(\cdot)+\ldots+f\left(v_{r}\right) \psi_{v_{r}}(\cdot)$ is harmonic, and agrees with $f$ on $S_{0}$. Therefore $S_{0} f=\phi$.

For the second statement, observe that $\psi_{v_{1}}(x)+\ldots+\psi_{v_{r}}(x)$ is the harmonic extension of a function which is identically equal to 1 on $V^{(0)}$. Because of its uniqueness, it must be identically equal to 1 on $\mathcal{K}$.

By self-similarity, this statement carries over to subsequent steps. More precisely, we have:

Corollary 3.1. Suppose that $\xi \in \mathcal{V}^{(m)}$, let $\Delta \in \mathcal{T}_{m-1}$ be the $(m-1)$-simplex $\xi$ belongs to, and let $\tilde{v}_{1}, \ldots, \tilde{v}_{r}$ be its vertices. Then

$$
S_{m-1} f(\xi)=f\left(\tilde{v}_{1}\right) \psi_{\tilde{v}_{1}}(\xi)+\cdots+f\left(\tilde{v}_{r}\right) \psi_{\tilde{v}_{r}}(\xi),
$$

with $\psi_{\tilde{v}_{1}}(x)+\ldots+\psi_{\tilde{v}_{r}}(x)=1$ for all $x \in \Delta$.

3.1. Characterization of Hölder-Zygmund spaces. We start with the characterization of the spaces $\Lambda_{\alpha}^{(1)}(\mathcal{K})$.

Theorem 3.1. Suppose the harmonic expansion of a function $f \in C(\mathcal{K})$ is given by (2.6), take $\alpha \in(0,1)$. Then $f \in \Lambda_{\alpha}^{(1)}(\mathcal{K})$ if and only if there exists a constant $M$ such that for $\xi \in \mathcal{V}^{(m)}$ one has

$$
\left|c_{\xi}\right| \leq M L^{-m \alpha\left(d_{w}-d_{f}\right)} .
$$

Proof. Suppose $f \in \Lambda_{\alpha}^{(1)}(\mathcal{K})$. Let $\xi \in \mathcal{V}^{(m)}$ and let $\Delta$ be the $(m-1)$-simplex $\xi$ belongs to, $\tilde{v}_{1}, \ldots, \tilde{v}_{r}$-its vertices. Then, by Corollary 3.1

$$
\begin{aligned}
\left|c_{\xi}\right| & =\left|f(\xi)-S_{m-1} f(\xi)\right|=\left|f(\xi)-\left(f\left(\tilde{v}_{1}\right) \psi_{\tilde{v}_{1}}(\xi)+\cdots+f\left(\tilde{v}_{r}\right) \psi_{\tilde{v}_{r}}(\xi)\right)\right| \\
& \leq \psi_{\tilde{v}_{1}}(\xi)\left|f(\xi)-f\left(\tilde{v}_{1}\right)\right|+\cdots+\psi_{\tilde{v}_{r}}(\xi)\left|f(\xi)-f\left(\tilde{v}_{r}\right)\right| .
\end{aligned}
$$

Points $\xi$ and $\tilde{v}_{k}$ might, or might not, be $m$-neighbors. If they are not, we find a chain $\xi=x_{0}, \ldots, x_{n}=\tilde{v}_{k}$ such that $x_{i} \in \Delta$ and $x_{i-1} \stackrel{m}{\sim} x_{i}, 1=1, \ldots, n-1$. It is clear that $n \leq r$, the number of all vertices of $\Delta$. In either case, we have

$$
\left|f(\xi)-f\left(\tilde{v}_{r}\right)\right| \leq\left|f\left(x_{0}\right)-f\left(x_{1}\right)\right|+\cdots+\left|f\left(x_{n-1}\right)-f\left(x_{n}\right)\right| \leq r \sup \{|f(x)-f(y)|: x \stackrel{m}{\sim} y\},
$$

so that

$$
\left|c_{\xi}\right| \leq \sup \{|f(x)-f(y)|: x \stackrel{m}{\sim} y\} \leq r M L^{-m \alpha\left(d_{w}-d_{f}\right)} .
$$

To get the opposite implication, suppose that the coefficients $c_{\xi}$ satisfy (3.3). 
Take $x \stackrel{m}{\sim} y$, then in particular $x, y \in V^{(m)}$, so that $f(x)=S_{m} f(x)$ and $f(y)=$ $S_{m} f(y)$. Therefore, using the assumption (3.3),

$$
\begin{aligned}
|f(x)-f(y)| & =\left|S_{m} f(x)-S_{m} f(y)\right|=\left|\sum_{i=0}^{m} \sum_{\xi \in \mathcal{V}^{(i)}} c_{\xi}\left(\psi_{\xi}(x)-\psi_{\xi}(y)\right)\right| \\
& \leq M \sum_{i=0}^{m} L^{-i \alpha\left(d_{w}-d_{f}\right)} \sum_{\xi \in \mathcal{V}^{(i)}}\left|\psi_{\xi}(x)-\psi_{\xi}(y)\right| .
\end{aligned}
$$

Because of the nesting, the self-similarity of the fractal, and the way the $m$-harmonic function arises, we infer that when $x \stackrel{m}{\sim} y, i \leq m, \xi \in \mathcal{V}^{(i)}$ then $\left|\psi_{\xi}(x)-\psi_{\xi}(y)\right|=$ $\left|\psi_{\bar{\xi}}(\bar{x})-\psi_{\bar{\xi}}(\bar{y})\right|$, for some $\bar{\xi} \in \mathcal{V}^{(0)}\left(=V^{(0)}\right)$ and $\bar{x} \stackrel{m-i}{\sim} \bar{y}$. The function $\psi_{\bar{\xi}}$ is 0 harmonic, i.e. harmonic, so by Proposition 5.12. of [13] it is Lipschitz with respect to the resistance metric $R(\cdot, \cdot)$ on $\mathcal{K}$. Therefore

$$
\left|\psi_{\xi}(x)-\psi_{\xi}(y)\right| \leq A(\bar{\xi}) R(\bar{x}, \bar{y})
$$

where $\bar{\xi}, \bar{x}, \bar{y}$ are as above. There are finitely many points $\bar{\xi}$ in $V^{(0)}$, so that $\sup _{\bar{\xi} \in V(0)} A(\bar{\xi})=: A<\infty$. Since the points $\bar{x}, \bar{y}$ are $(m-i)$-neighbors, there exists $w=\left(i_{1}, \ldots, i_{m-i}\right)$ such that $\bar{x}=\psi_{w}\left(v_{1}\right), \bar{y}=\psi_{w}\left(v_{2}\right)$, for certain $v_{1}, v_{2} \in V^{(0)}$. By Theorem A.1. of [13], one has

$$
\begin{aligned}
R(\bar{x}, \bar{y}) & =R\left(\psi_{w}\left(v_{1}\right), \psi_{w}\left(v_{2}\right)\right) \leq \rho^{-(m-i)} R\left(v_{i}, v_{2}\right) \\
& \leq \rho^{-(m-i)} \sup _{v_{1}, v_{2} \in V^{(0)}} R\left(v_{i}, v_{2}\right)=C \rho^{-(m-i)},
\end{aligned}
$$

where $\rho$ is the resistance scaling factor from Theorem 2.1 above. Note that $\rho=$ $L^{d_{w}-d_{f}}$. Collecting all of these, we see that

$$
\left|\psi_{\xi}(x)-\psi_{\xi}(y)\right| \leq A \cdot C \cdot L^{-(m-i)\left(d_{w}-d_{f}\right)} .
$$

According to Lemma 3.1, when $x, y \in \mathcal{V}^{(m)}$ are given, there are at most $2 r$ vertices $\xi \in \mathcal{V}^{(i)}$ for which the difference $\left|\psi_{\xi}(x)-\psi_{\xi}(y)\right|$ might be nonzero, so that finally

$$
|f(x)-f(y)| \leq 2 r M A C \sum_{i=0}^{m} L^{-i \alpha\left(d_{w}-d_{f}\right)} L^{-(m-i)\left(d_{w}-d_{f}\right)} \leq C(\mathcal{K}, \alpha) L^{-m \alpha\left(d_{w}-d_{f}\right)} .
$$

The constant in the last expression depends on on the geometry of $\mathcal{K}$ and on $\alpha$ (it explodes as $\alpha \rightarrow 1$ ). The theorem is proven.

3.2. Characterization of Besov-Lipschitz spaces. We now will give a characterization of functions from $\left(\Lambda_{\alpha}^{p, q}\right)^{(1)}(\mathcal{K})$, similar to that from [7], but expressed in terms of their coefficients in the harmonic basis.

Theorem 3.2. Let $\mathcal{K}$ be a nested fractal, and $f$ a continuous function on $\mathcal{K}$ having the harmonic expansion (2.6). Suppose $1 \leq p, q \leq \infty, \alpha>\frac{d}{p}$, and assume that $\psi_{v_{1}} \in\left(\Lambda_{\beta}^{p, \infty}\right)^{(1)}(\mathcal{K})$ for some $\beta>\alpha$, where $v_{1}$ is an arbitrarily chosen vertex from $V^{(0)}$, and $\psi_{v_{1}}$ is a harmonic function from Definition 2.4. Then $f \in\left(\Lambda_{\alpha}^{p, q}\right)^{(1)}(\mathcal{K})$ if and only if $S_{\alpha}^{p, q}(f)<\infty$, where $(1 \leq p, q<\infty)$

$$
S_{\alpha}^{p, q}(f):=\left(\sum_{m=0}^{\infty} L^{\left(d_{w}-d_{f}\right) \alpha m q}\left(\frac{1}{L^{m d_{f}}} \sum_{\xi \in \mathcal{V}^{(m)}}\left|c_{\xi}\right|^{p}\right)^{q / p}\right)^{1 / q}<\infty .
$$


Moreover, we have

$$
\frac{1}{C}\|f\|_{p, q, \alpha} \leq S_{\alpha}^{p, q}(f) \leq C\|f\|_{p, q, \alpha},
$$

where $C>0$ is a universal constant, not depending on $f$. (When $p=\infty$ or $q=\infty$, then the usual modifications are needed.)

Remark 3.1. For the interval $[0,1]$ Th. 3.2 gives the same characterization of $B_{p, q}^{\alpha}(0,1)$ as Th. 5.1 in [7] since the harmonic functions on the unit interval are precisely the linear functions, $B_{p, q}^{\alpha}(\mathcal{K})=\operatorname{Lip}(\alpha, p, q, \mathcal{K})$ for $0<\alpha<1$, and $\operatorname{Lip}(\alpha, p, q, \mathcal{K})=\left(\Lambda_{\alpha /\left(d_{w}-d_{f}\right)}^{p, q}\right)^{(1)}(\mathcal{K})$ for $\alpha>d_{f} / p$. However, on the Sierpiński gasket $\mathcal{G}$ and small $p$, Th. 3.2 provides a characterization of $\operatorname{Lip}(\alpha, p, q, \mathcal{G})$ for higher smoothness $\alpha \geq 1$ than with a piecewise linear base, since a piecewise linear function does not belong to $\left(\Lambda_{\alpha /\left(d_{w}-d_{f}\right)}^{p, \infty}\right)^{(1)}(\mathcal{G})$ for $\alpha>1$. On the other hand, for large $p$, since a harmonic function belong to $\Lambda_{\alpha /\left(d_{w}-d_{f}\right)}^{(1)}(\mathcal{G})$ iff $\alpha /\left(d_{w}-d_{f}\right) \leq 1$, it follows from the embedding (Lemma 4.2 of [21])

$$
\left(\Lambda_{\sigma}^{p, q}\right)^{(1)}(\mathcal{G}) \subseteq \Lambda_{\sigma-d_{f} /\left(p\left(d_{w}-d_{f}\right)\right)}^{(1)}(\mathcal{G}),
$$

with $\sigma=\alpha /\left(d_{w}-d_{f}\right)$, that a piecewise harmonic function does not belong to $\left(\Lambda_{\alpha /\left(d_{w}-d_{f}\right)}^{p, q}\right)^{(1)}(\mathcal{G})$ for $\alpha>d_{f} / p+\left(d_{w}-d_{f}\right)$, suggesting that using either of the bases has its limitations.

Proof. For short, denote $R_{m, p}(f)=\left(\frac{1}{L^{m d_{f}}} \sum_{\xi \in \mathcal{V}^{(m)}}\left|c_{\xi}\right|^{p}\right)^{1 / q}$, and

$$
e_{m}(f)=L^{\left(d_{w}-d_{f}\right) \alpha m} R_{m, p}(f), \text { for } m=0,1,2, \ldots
$$

In this notation, one has $S_{\alpha}^{p, q}(f)=\left\|\left(e_{m}(f)\right)\right\|_{q}$.

Part 1. Assume that $f \in\left(\Lambda_{\alpha}^{p, q}\right)^{(1)}(\mathcal{K})$, i.e. $\left\|\left(L^{\left(d_{w}-d_{f}\right) \alpha m} \delta_{m, p}(f)\right)\right\|_{q}<\infty$. We will show that

$$
R_{m, p}(f) \leq C \delta_{m, p}(f),
$$

with $C>0$ not depending on $m, f$. This inequality will result in

$$
e_{m}(f) \leq L^{\left(d_{w}-d_{f}\right) \alpha m} \delta_{m, p}(f)
$$

and consequently we will get

$$
S_{\alpha}^{p, q}(f)<C\|f\|_{p, q, \alpha}<\infty .
$$

To prove (3.6), take $\xi \in \mathcal{V}^{(m)}$, and assume that $\Delta \in \mathcal{T}_{m-1}$ is such that $\xi \in$ $\mathcal{V}^{(m)} \cap \Delta$. There is only one such $\Delta$ (this is because $(m-1)$-cells meets through points from $V^{(m-1)}$ by nesting, and $\left.\xi \notin V^{(m-1)}\right)$. Let $w=\left(i_{1}, \ldots, i_{m-1}\right)$ be the address of the cell $\Delta$, i.e. $\Delta=\phi_{w}(\mathcal{K})$, and let $\tilde{v}_{1}, \ldots, \tilde{v}_{r}$ be the vertices of $\Delta$. It follows that there exist $k \in\{1, \ldots, M\}, l \in\{1, \ldots, r\}$ such that

$$
\xi=\phi_{w}\left(\phi_{k}\left(\tilde{v}_{l}\right)\right) \text {. }
$$

To estimate $\left|c_{\xi}\right|$, we use Lemma 3.2, or rather its Corollary 3.1. We can write

$$
\begin{aligned}
c_{\xi} & =f(\xi)-S_{m-1} f(\xi)=f(\xi)-\left(f\left(\tilde{v}_{1}\right) \psi_{\tilde{v}_{1}}(\xi)+\cdots+f\left(\tilde{v}_{r}\right) \psi_{\tilde{v}_{r}}(\xi)\right) \\
& =\psi_{\tilde{v}_{1}}(\xi)\left(f(\xi)-f\left(\tilde{v}_{1}\right)\right)+\ldots+\psi_{\tilde{v}_{r}}(\xi)\left(f(\xi)-f\left(\tilde{v}_{r}\right)\right) .
\end{aligned}
$$


Jensen's inequality gives

$$
\left|c_{\xi}\right|^{p} \leq \psi_{\tilde{v}_{1}}(\xi)\left|f(\xi)-f\left(\tilde{v}_{1}\right)\right|^{p}+\cdots+\psi_{\tilde{v}_{r}}(\xi)\left|f(\xi)-f\left(\tilde{v}_{r}\right)\right|^{p} .
$$

If $\xi$ and $\tilde{v}_{k}$ belong to a common $m$-cell, we do nothing and leave $\left|f(\xi)-f\left(\tilde{v}_{k}\right)\right|$ as it is.

Otherwise, we can find a chain $\xi=x_{0}, x_{1}, \ldots, x_{n}=\tilde{v}_{k}$ such that $x_{j} \in V^{(m)}$ and $x_{j-1} \stackrel{m}{\sim} x_{j}, j=1, \ldots, n$. The length of this chain is less than or equal to $r$. In this case, we have, using the elementary inequality $\left(y_{1}+\cdots+y_{n}\right)^{p} \leq n^{p-1}\left(y_{1}^{p}+\cdots+y_{n}^{p}\right)$ :

$$
\begin{aligned}
\left|f(\xi)-f\left(\tilde{v}_{k}\right)\right|^{p} & \leq\left(\left|f(\xi)-f\left(x_{1}\right)\right|+\cdots+\left|f\left(x_{n-1}\right)-f\left(\tilde{v}_{k}\right)\right|\right)^{p} \\
& \leq n^{p-1}\left(\left|f(\xi)-f\left(x_{1}\right)\right|^{p}+\cdots\left|f\left(x_{n-1}\right)-f\left(\tilde{v}_{k}\right)\right|^{p}\right) \\
& \leq r^{p-1} \sum_{x, y \in \Delta, x_{\sim}^{m} y}|f(x)-f(y)|^{p},
\end{aligned}
$$

and hence

$$
\left|c_{\xi}\right|^{p} \leq r^{p-1} \sum_{x, y \in \Delta, x \sim y}|f(x)-f(y)|^{p} .
$$

Since the number of points from $\mathcal{V}^{(m)}$ in any $\Delta \in \mathcal{T}_{m-1}$ is fixed, summing up we arrive at

$$
\sum_{\xi \in \mathcal{V}^{(m)} \cap \Delta}\left|c_{\xi}\right|^{p} \leq C \sum_{\substack{m \\ \sim} y, x, y \in \Delta}|f(x)-f(y)|^{p},
$$

where the constant $C$ does not depend on $f$ nor on $m$. Because of the nesting, the constant $C$ is the same for all $m \geq 1$ and all $(m-1)$-cells $\Delta$. This way we obtain

$$
\begin{aligned}
\sum_{\xi \in \mathcal{V}^{(m)}}\left|c_{\xi}\right|^{p} & \leq \sum_{\Delta \in \mathcal{T}_{m-1}} \sum_{\xi \in \mathcal{V}^{(m)} \cap \Delta}\left|c_{\xi}\right|^{p} \leq \sum_{\Delta \in \mathcal{T}_{m-1}} C \sum_{\substack{m \sim m \\
x \sim x, y \in \Delta}}|f(x)-f(y)|^{p} \\
& \leq C \sum_{\substack{m \\
x \sim y}}|f(x)-f(y)|^{p} .
\end{aligned}
$$

Therefore (3.6) is proven.

Part 2. We now assume that $f \in L^{p}(\mathcal{K})$ is such that $S_{\alpha}^{p, q}(f)<+\infty$. From the Minkowski inequality and the definition of $\delta_{p, m}$ we have

$$
\begin{aligned}
\delta_{m, p}(f) & =\delta_{m, p}\left(\sum_{i=0}^{m} \sum_{\xi \in \mathcal{V}^{(i)}} c_{\xi} \psi_{\xi}\right) \leq \sum_{i=0}^{m} \delta_{p, m}\left(\sum_{\xi \in \mathcal{V}^{(i)}} c_{\xi} \psi_{\xi}\right) \\
& =\sum_{i=0}^{m}\left(\frac{1}{L^{m d_{f}}} \sum_{\substack{m \\
\sim}}\left|\sum_{\xi \in \mathcal{V}^{(i)}} c_{\xi}\left(\psi_{\xi}(x)-\psi_{\xi}(y)\right)\right|^{\frac{1}{p}}\right. \\
& \leq \sum_{i=0}^{m}\left(\frac{1}{L^{m d_{f}}}(2 r)^{p-1} \sum_{\xi \in \mathcal{V}^{(i)}}\left|c_{\xi}\right|^{p} \sum_{x \sim y}\left|\psi_{\xi}(x)-\psi_{\xi}(y)\right|^{p}\right)^{1 / p},
\end{aligned}
$$

where the last inequality follows from the fact that, given $x \stackrel{m}{\sim} y$, there are at most $2 r$ nonvanishing terms in the sum $\sum_{\xi \in \mathcal{V}^{(i)}} c_{\xi}\left(\psi_{\xi}(x)-\psi_{\xi}(y)\right)$, for every $i \leq m$ (see Lemma 3.1). 
Next, from the scaling and symmetry, we get, when $i \leq m$ and $\xi \in \mathcal{V}^{(i)}$ are given,

$$
\sum_{\substack{m \\ x \sim y}}\left|\psi_{\xi}(x)-\psi_{\xi}(y)\right|^{p} \leq r \sum_{\substack{m-i \\ x^{m} \sim}}\left|\psi_{\bar{\xi}}(x)-\psi_{\bar{\xi}}(y)\right|^{p},
$$

for some $\bar{\xi} \in \mathcal{V}^{(0)}$. We will estimate the last sum using the assumption $\psi_{\bar{\xi}} \in\left(\Lambda_{\beta}^{p, \infty}\right)^{(1)}$. Indeed, since

$$
\sup _{n} L^{\left(d_{w}-d_{f}\right) \beta n} \delta_{n, p}\left(\psi_{\bar{\xi}}\right)=: B \leq \infty
$$

it follows that

$$
\sum_{\substack{n \\ x \sim y}}\left|\psi_{\bar{\xi}}(x)-\psi_{\bar{\xi}}(y)\right|^{p} \leq B^{p} L^{-\left(d_{w}-d_{f}\right) n p \beta} L^{n d_{f}} .
$$

Inserting (3.8) and (3.9) into (3.7) we see that

$$
\delta_{p, m}(f) \leq C L^{-\left(d_{w}-d_{f}\right) \beta m} \sum_{i=0}^{m}\left(\frac{L^{i\left(d_{w}-d_{f}\right) p \beta}}{L^{i d_{f}}} \sum_{\xi \in \mathcal{V}^{(i)}}\left|c_{\xi}\right|^{p}\right)^{1 / p} .
$$

Therefore,

$$
\sum_{m=0}^{\infty} L^{\left(d_{w}-d_{f}\right) \alpha m q}\left(\delta_{p, m}(f)\right)^{q} \leq C \sum_{m=1}^{\infty} L^{\left(d_{w}-d_{f}\right)(\alpha-\beta) m q}\left(\sum_{i=0}^{m} a_{i}^{1 / p}\right)^{q},
$$

where we have denoted $a_{i}=L^{i\left(d_{w}-d_{f}\right) p \beta-i d_{f}} \sum_{\xi \in \mathcal{V}^{(i)}}\left|c_{\xi}\right|^{p}$. Since $(\alpha-\beta)<0$, we can implement the classical discrete Hardy inequality

$$
\sum_{m=1}^{\infty} \rho^{m}\left(\sum_{i=0}^{m} x_{i}\right)^{q} \leq K(\rho, q) \sum_{m=1}^{\infty} \rho^{m} x_{m}^{q}
$$

valid for $\rho \in(0,1), q>0, x_{m}>0$ (with the convention: left-hand side infinite implies right-hand side infinite). We obtain

$$
\begin{aligned}
\sum_{m=0}^{\infty} L^{\left(d_{w}-d_{f}\right) \alpha m q}\left(\delta_{p, m}(f)\right)^{q} & \leq K \sum_{m=0}^{\infty} L^{\left(d_{w}-d_{f}\right)(\alpha-\beta) q m} a_{m}^{q / p} \\
& =K \sum_{m=0}^{\infty} L^{\left(d_{w}-d_{f}\right) \alpha q m}\left(\frac{1}{L^{m d_{f}}} \sum_{\xi \in \mathcal{V}^{(m)}}\left|c_{\xi}\right|^{p}\right)^{q / p} \\
& =K \sum_{m=0}^{\infty} L^{\left(d_{w}-d_{f}\right) \alpha q m} R_{m, p}(f)=K\left[S_{\alpha}^{p, q}(f)\right]^{q}
\end{aligned}
$$

We are done.

We would like to discuss the importance of the assumption $\psi_{v_{1}} \in\left(\Lambda_{\beta}^{p, \infty}\right)^{(1)}(\mathcal{K})$. When $p=2$, the answer is complete: all $m$-harmonic functions belong to the domain of the Brownian-motion induced Dirichlet form on $\mathcal{K}$, which is known (see [16]) to be $\operatorname{Lip}\left(\frac{d_{w}}{2}, 2, \infty, \mathcal{K}\right)=\left(\Lambda_{\beta}^{2, \infty}\right)^{(1)}(\mathcal{K})$, with $\beta=\frac{d_{w}}{2\left(d_{w}-d_{f}\right)}$. Also, we know that the spaces $\operatorname{Lip}(\gamma, 2, \infty, \mathcal{K})$ are trivial for $\gamma>\frac{d_{w}}{2}$, only containing constant functions; therefore Theorem 3.2 is valid for for $\frac{d}{2}=\frac{d_{f}}{2\left(d_{w}-d_{f}\right)}<\alpha<\frac{d_{w}}{2\left(d_{w}-d_{f}\right)}$. Since on nested fractals one always has $d_{f}<d_{w}$ (see [1], Corollary 3.12), this interval is nonempty for any nested fractal $\mathcal{K}$. 


\subsection{Additional remarks.}

Remark 3.2. It is usually very hard to point out any concrete function that belongs to the Besov-Lipschitz space $\left(\Lambda_{\alpha}^{p, q}\right)^{(1)}(\mathcal{K})$. Even in the best-analyzed case $p=2$, $q=\infty, \alpha=\frac{d_{w}}{2\left(d_{w}-d_{f}\right)}$, giving an actual example of a function in this space is not easy. This space coincides with the domain $\mathcal{D}(\mathcal{E})$ of the Dirichlet form for the Brownian motion on $\mathcal{K}$, and from its definition we immediately obtain that all the $m$-harmonic function on $\mathcal{K}$ belong to that space. For the Sierpiński gasket $\mathcal{G}$ it is known that if a function belongs to $\mathcal{D}(\mathcal{E})$ and has a $C^{1}$-extension to a vicinity of $\mathcal{G}$, then it must be constant. Also, by probabilistic arguments we know that the transition density of the Brownian motion on $\mathcal{K}, p(t, x, \cdot)$ belongs to $\mathcal{D}(\mathcal{E})$.

Now, using our Theorem 3.2 we can give nontrivial examples of functions in $\mathcal{D}(\mathcal{E})$.

Let us work with the 2-dimensional gasket $\mathcal{G}$. There are $3^{m}$ points in $\mathcal{V}^{(m)}$, and so by setting $c_{\xi}=\sqrt{5^{-m}}$ for $\xi \in \mathcal{V}^{(m)}, f(x)=\sum_{m} \sqrt{5^{-m}} \sum_{\xi \in \mathcal{V}^{(m)}} \psi_{\xi}(x)$, we get a function that belongs to $\mathcal{D}(\mathcal{E})$. Also, we can infer that taking $c_{\xi}=a_{m} \sqrt{5^{-m}}$ for $\xi \in \mathcal{V}^{(m)}$, with $a_{m} \rightarrow \infty$, results in a function that is not in $\mathcal{D}(\mathcal{E})$.

Remark 3.3. The spaces $\left(\Lambda_{\alpha}^{p, q}\right)^{(1)}$ are infinite dimensional only for small values of $\alpha$, since it follows from the definition that there exists a threshold $\alpha_{p, q}$ such that the spaces $\left(\Lambda_{\alpha}^{p, q}\right)^{(1)}$ consist of constant functions for $\alpha>\alpha_{p, q}$. Its precise value is know only for specific values of parameters. It was proven in [17] that for any nested fractal $\mathcal{K}$, the spaces $\operatorname{Lip}(\alpha, p, p, \mathcal{K})$ with $p \geq 2$ degenerate and consist of constant functions only for $\alpha \geq \frac{d_{w}}{2}$. Because of the inclusions

$$
\operatorname{Lip}\left(\alpha^{\prime}, p, \infty, \mathcal{K}\right) \subset \operatorname{Lip}(\alpha, p, p, \mathcal{K}) \subset \operatorname{Lip}(\alpha, p, \infty, \mathcal{K}), \quad \alpha^{\prime} \geq \alpha,
$$

and the relation $\operatorname{Lip}(\alpha, p, q, \mathcal{K})=\left(\Lambda_{\alpha / d_{w}-d_{f}}^{p, q}\right)^{(1)}(\mathcal{K})$ valid for $\alpha>d_{f} / p$, we get that when $p \geq 2, \alpha_{p, p}(\mathcal{K}) \leq \frac{d_{w}}{2\left(d_{w}-d_{f}\right)}$ and $\alpha_{p, \infty}(\mathcal{K}) \leq \frac{d_{w}}{2\left(d_{w}-d_{f}\right)}$ as well. For $p=2$ we have equalities here, as $\operatorname{Lip}\left(\frac{d_{w}}{2}, 2, \infty, \mathcal{K}\right)$ and $\operatorname{Lip}\left(\alpha \frac{d_{w}}{4}, 2,2, \mathcal{K}\right)$ are the domains of the Brownian motion and the $\alpha$-stable processes on $\mathcal{K}, \alpha<2$, respectively, and as such are infinite-dimensional (dense, in fact) subspaces of $L^{2}(\mathcal{K})$. See [16], [19].

Harmonic functions provide means for finding bounds for $\alpha_{p, q}$ : if $\psi_{\xi} \in\left(\Lambda_{\beta}^{p, q}\right)^{(1)}$ for one (all) $\xi \in V^{(0)}$, then all spaces $\left(\Lambda_{\alpha}^{p, q}\right)^{(1)}$ for $d / p<\alpha<\beta$ are nontrivial (in fact infinite-dimensional) and $\alpha_{p, q} \geq \beta$. In Example 3.1 below we use this to show that on the Sierpiński gasket, $\alpha_{p, \infty}(\mathcal{G})>\frac{d_{w}}{2\left(d_{w}-d_{f}\right)}$ for $p \leq 2$.

Example 3.1. In this example we give a lower bound for $\alpha_{p, \infty}$ on the Sierpiński gasket $\mathcal{G}$ by using interpolation and harmonic functions. Let $\beta_{p, q}$ denote the upper bound for when a harmonic function belong to $\left(\Lambda_{\alpha}^{p, q}\right)^{(1)}(\mathcal{G})$. Note that we may have $\alpha_{p, q}>\beta_{p, q}$, which is the case for $p=q=\infty$. Finding $\beta_{p, \infty}$ is of particular interest to determine when we can apply Theorem 3.2.

A harmonic function $\psi$ belongs to $\left(\Lambda_{\alpha}^{p, \infty}\right)^{(1)}(\mathcal{G})$ if

$$
\delta_{m, p}(\psi) \leq c 2^{-\left(d_{w}-d_{f}\right) \alpha m} \quad \text { for all } m \geq 0 .
$$

For $1 \leq p_{0} \leq p \leq p_{1} \leq \infty$, it follows from the inequality $\left\|a_{m}\right\|_{l^{p}} \leq\left\|a_{m}\right\|_{l^{p_{0}}}^{(1-\theta)}\left\|a_{m}\right\|_{l^{p_{1}}}^{\theta}$ that

$$
\delta_{m, p}(\psi) \leq\left(\delta_{m, p_{0}}(\psi)\right)^{(1-\theta)}\left(\delta_{m, p_{1}}(\psi)\right)^{\theta},
$$

where $\theta$ is given by $1 / p=(1-\theta) / p_{0}+\theta / p_{1}$. 
If we use that $\beta_{2, \infty}=\frac{d_{w}}{2\left(d_{w}-d_{f}\right)}$ and $\beta_{\infty, \infty}=1$ on the Sierpinski gasket, and combine (3.11) with (3.12) for $p_{0}=2$ and $p_{1}=\infty$, we get that

$$
\delta_{m, p}(\psi) \leq c_{p} 2^{-m\left(d_{w}-d_{f}\right)\left(d_{w} /\left(d_{w}-d_{f}\right)+p-2\right) / p} .
$$

Therefore $\psi \in\left(\Lambda_{\alpha}^{p, \infty}\right)^{(1)}(\mathcal{G})$, with $\alpha=\left(d_{w} /\left(d_{w}-d_{f}\right)+p-2\right) / p$, and hence $\beta_{p, \infty} \geq$ $\left.d_{w} /\left(d_{w}-d_{f}\right)+p-2\right) / p$ for $p \geq 2$.

Next we proceed by interpolating between $p=1$ and $p=2$. However, since we do not know the actual value of $\beta_{1, \infty}$ we will first give an estimate on the form (3.11) for $p=1$. A harmonic function $\psi$ is completely determined by its values on $V^{(0)}$. More precise, for a point $q \in V^{(m)} \backslash V^{(m-1)}, \psi(q)$ is a weighted average of its values over the three vertices in the $m-1$-simplex it belongs to (see [21] for details)

$$
\psi(q)=\frac{2}{5} \psi\left(p_{1}\right)+\frac{2}{5} \psi\left(p_{2}\right)+\frac{1}{5} \psi\left(p_{3}\right) .
$$

The weights here are specific for the Sierpiński gasket, but a similar expression can be derived for any nested fractal. We can use (3.13) three times to write the difference $\psi(x)-\psi(y)$ for $x \stackrel{m}{\sim} y$ as the sum of differences $\psi(\bar{x})-\psi(\bar{y})$ for $\bar{x} \stackrel{m-3}{\sim} \bar{y}$. Then, by using the triangle inequality and summing over all $m$-neighbors we get that $\delta_{m, 1}(\psi) \leq$ $c 2^{-\left(d_{w}-d_{f}\right)\left(d_{f} /\left(d_{w}-d_{f}\right)+\beta_{3}\right) m}$, where $\beta_{3}=\log \left(268 / 5^{3}\right) /(3 \log (3 / 5))$, and $c$ is a positive constant. Therefore, applying (3.12) with $p_{0}=1$ and $p_{1}=2$, we get

$$
\delta_{m, p}(\psi) \leq c_{p} 2^{-\left(d_{w}-d_{f}\right)\left(\left(\beta_{3}+d_{f} /\left(d_{w}-d_{f}\right)\right)(2-p)+(p-1)\left(d_{f} /\left(d_{w}-d_{f}\right)+1\right)\right) / p m},
$$

which implies that $\psi \in\left(\Lambda_{\alpha}^{p, \infty}\right)^{(1)}(\mathcal{G})$, where $\alpha=\left(d_{f} /\left(d_{w}-d_{f}\right)+p-1+\beta_{3}(2-p)\right) / p$. Hence, for $1 \leq p \leq 2$, we have that

$$
\beta_{p, \infty} \geq\left(d_{w} /\left(d_{w}-d_{f}\right)+p-2+\beta_{3}(2-p)\right) / p,
$$

since $d_{f} /\left(d_{w}-d_{f}\right)+1=d_{w} /\left(d_{w}-d_{f}\right)$. Therefore

$$
\alpha_{p, \infty}(\mathcal{G}) \geq \beta_{p, \infty} \geq \begin{cases}\left(d_{w} /\left(d_{w}-d_{f}\right)+p-2+\beta_{3}(2-p)\right) / p, & 1 \leq p<2, \\ \left(d_{w} /\left(d_{w}-d_{f}\right)+p-2\right) / p, & 2<p \leq \infty\end{cases}
$$

on the Sierpiński gasket. It follows that for small $p$, Theorem 3.2 gives a characterization of $\operatorname{Lip}(\alpha, p, q, \mathcal{G})$ for some $\alpha \geq 1$.

And for $p \geq 2$, we get matching bounds for $\alpha_{p, \infty}(\mathcal{G})$

$$
\left(d_{w} /\left(d_{w}-d_{f}\right)+p-2\right) / p \leq \alpha_{p, \infty}(\mathcal{G}) \leq d_{w} /\left(2\left(d_{w}-d_{f}\right)\right) .
$$

For $p=2$ these two bounds coincide, but for $p \neq 2$ the question what is the actual threshold remains open, even for the Sierpiński gasket.

Acknowledgement. The authors would like to thank Professor Robert S. Strichartz for valuable comments and suggestions.

\section{References}

[1] Barlow, M. T.: Diffusion on fractals. - Lecture Notes in Math. 1690, Lectures on Probability and Statistics, Ecole d'Eté de Prob. de St. Flour XXV (1995), Springer-Verlag, Berlin, 1998.

[2] Bodin, M.: Discrete characterisations of Lipschitz spaces on fractals. - Math. Nachr. 282:1, 2009, 26-43.

[3] Falconer, K.: Geometry of fractal sets. - Cambridge Univ. Press, Cambridge, 1985. 
[4] Grigoryan, A., J. Hu, and K.S. LaU: Heat kernels on metric measure spaces and an application to semilinear elliptic equations. - Trans. Amer. Math. Soc. 355, 2003, 2065-2095.

[5] Jonsson, A.: Brownian motion on fractals and function spaces. - Math. Z. 222, 1996, 495-504.

[6] Jonsson, A.: Triangulations of closed sets and bases in function spaces. - Ann. Acad. Sci. Fenn. Math. 29:1, 2004, 43-58.

[7] Jonsson, A., and A. Kamont: Piecewise linear bases and Besov spaces on fractal sets. Anal. Math. 27:2, 2001, 77-117.

[8] Jonsson, A., and H. Wallin: Function spaces on subsets on $\mathbf{R}^{n}$. - Math. Rep. 2:1, 1984.

[9] Kabanava, M.: Characterization of besov spaces on nested fractals by piecewise harmonic functions. - Z. Anal. Anwend. 31:2, 2012, 183-201.

[10] Kigami, J.: A harmonic calculus on the Sierpiński spaces. - Japan J. Appl. Math. 6, 1989, 259-290.

[11] Kigami, J.: Harmonic calculus on p.c.f. self-similar sets. - Trans. Amer. Math. Soc. 335, 1993, $721-755$.

[12] Kigami, J.: Analysis on fractals. - Cambridge Tracts in Math. 143, Cambridge Univ. Press, Cambridge, 2001.

[13] Kigami, J.: Harmonic analysis for resistance forms. - J. Funct. Anal. 204, 2003, 399-444.

[14] Kusuoka, S.: Dirichlet forms on fractals and products of random matrices. - Publ. Res. Inst. Math. Sci. 25, 1989, 659-680.

[15] Lindstrøm, T.: Brownian motion on nested fractals. - Mem. Amer. Math. Soc. 420, 1990.

[16] Pietruska-Paluba, K.: Some function spaces related to the Brownian motion on simple nested fractals. - Stochastics 67:3-4, 1999, 267-285.

[17] Pietruska-Paluba, K.: Heat kernels on metric spaces and a characterisation of constant functions. - Manuscripta Math. 115:3, 2004, 389-399.

[18] Saвот, C., Existence et unicité de la diffusion sur un ensemble fractal. - C. R. Acad. Sci. Paris Sér. I Math. 321:8, 1995, 1053-1059.

[19] Stos, A.: Symmetric $\alpha$-stable processes on $d$-sets. - Bull. Polish Acad. Sci. Math. 48:3, 2000, $237-245$.

[20] Strichartz, R. S.: Taylor approximations on Sierpiński gasket type fractals. - J. Funct. Anal. 174, 2000, 76-127.

[21] Strichartz, R. S.: Function spaces on fractals. - J. Funct. Anal. 198, 2003, 43-83.

[22] Teplyaev, A.: Gradients on fractals. - J. Funct. Anal. 174, 2000, 128-154. 